University of New Hampshire

University of New Hampshire Scholars' Repository

Space Science Center

Institute for the Study of Earth, Oceans, and

Space (EOS)

10-2007

\title{
Design optimization and performance capabilities of the fast neutron imaging telescope (FNIT)
}

\author{
U Bravar \\ University of New Hampshire - Main Campus \\ P J. Bruillard \\ University of New Hampshire - Main Campus \\ E O. Fluckiger \\ Universität Bern \\ Jason S. Legere \\ University of New Hampshire, jslegere@unh.edu \\ John R. Macri \\ University of New Hampshire - Main Campus, John.Macri@unh.edu
}

See next page for additional authors

Follow this and additional works at: https://scholars.unh.edu/ssc

Part of the Astrophysics and Astronomy Commons

\section{Recommended Citation \\ Bravar, U.; Bruillard, P.J.; Fluckiger, E.O.; Legere, J.S.; Macri, J.R.; MacKinnon, A.L.; Mallik, P.; McConnell, M.L.; Moser, M.R.; Pirard, B.; Ryan, J.M.; Woolf, R.S., "Design optimization and performance capabilities of the fast neutron imaging telescope (FNIT)," Nuclear Science Symposium Conference Record, 2007. NSS '07. IEEE , vol.1, no., pp.264,269, Oct. 26 2007-Nov. 32007}

This Conference Proceeding is brought to you for free and open access by the Institute for the Study of Earth, Oceans, and Space (EOS) at University of New Hampshire Scholars' Repository. It has been accepted for inclusion in Space Science Center by an authorized administrator of University of New Hampshire Scholars' Repository. For more information, please contact Scholarly.Communication@unh.edu. 


\section{Authors}

U Bravar, P J. Bruillard, E O. Fluckiger, Jason S. Legere, John R. Macri, A L. MacKinnon, Procheta Mallik, Mark L. McConnell, M R. Moser, B Pirard, James M. Ryan, and R S. Woolf 


\title{
Design Optimization and Performance Capabilities of the Fast Neutron Imaging Telescope (FNIT)
}

\author{
Ulisse Bravar, Paul J. Bruillard, Erwin O. Flückiger, Jason S. Legere, John R. Macri, Alec L. MacKinnon, Procheta \\ Mallik, Mark L. McConnell, Michael R. Moser, Benoit Pirard, James M. Ryan, and Richard S. Woolf
}

\begin{abstract}
We describe the design optimization process and performance characterization of a next generation neutron telescope, with imaging and energy measurement capabilities, sensitive to neutrons in the $1-20 \mathrm{MeV}$ energy range. The response of the Fast Neutron Imaging Telescope (FNIT), its efficiency in neutron detection, energy resolution and imaging capabilities were characterized through a combination of lab tests and Monte Carlo simulations. Monte Carlo simulations, together with experimental data, are also being used in the development and testing of the image reconstruction algorithm. FNIT was initially conceived to study solar neutrons as a candidate instrument for the Inner Heliosphere Sentinel (IHS) spacecraft. However, the design of this detector was eventually adapted to locate Special Nuclear Material (SNM) sources for homeland security purposes, by detecting fission neutrons. In either case, the detection principle is based on multiple elastic neutron-proton scatterings in organic scintillator. By reconstructing event locations and measuring the recoil proton energies, the direction and energy spectrum of the primary neutron flux can be determined and neutron sources identified. This paper presents the most recent results arising from our efforts and outlines the performance of the FNIT detector.
\end{abstract}

\section{INTRODUCTION}

THIS paper describes a compact Fast Neutron Imaging 1 Telescope (FNIT) for the measurement of neutrons in the 1-20 MeV energy range. The applications of FNIT are twofold. It was first conceived as a spacecraft-based detector to measure the neutron flux from solar flares in the inner heliosphere, to be deployed on either a NASA Solar Sentinel spacecraft [1] or on the Solar Orbiter mission under formulation at ESA [2]. In addition, the energy range and imaging capabilities of FNIT make it an ideal instrument for the identification of samples of Special Nuclear Material (SNM) through their neutron signature for homeland security purposes.

The FNIT concept evolved through a series of significantly different structural designs [3]. A full prototype science model was recently assembled at the University of New Hampshire (UNH) and underwent performance testing. The prototype was then calibrated at the pulsed neutron source at Crocker Nuclear Laboratory, UC Davis and exposed to a ${ }^{239} \mathrm{Pu}$ SNM sample at Pacific Northwest National Laboratory (PNNL).

\section{SOlAR NeUtrons}

Manuscript received November 23, 2007. This work was supported in part by the U.S. Department of Energy under Contracts DE-FG52-04NA25687 \& DE-FG52-06NA27493 and NASA under grant NAG5-13519 in the United States and the Swiss National Science Foundation under grant 200020$105435 / 1$ in Switzerland.

U. Bravar is with the Space Science Center, University of New Hampshire, Durham, NH 03824, USA (e-mail: ulisse.bravar@unh.edu).

P. J. Bruillard, formerly with the Space Science Center, University of New Hampshire, Durham, NH 03824, USA, is now with the Dept. of Physics, Texas A\&M University, College Station, TX 77843, USA.

E. O. Flückiger is with the Physikalisches Institut, University of Bern, CH-3012 Bern, Switzerland.

Jason S. Legere is with the Space Science Center, University of New Hampshire, Durham, NH 03824, USA.

J. R. Macri is with the Space Science Center, University of New Hampshire, Durham, NH 03824, USA.

A. L. MacKinnon is with the Dept. of Physics and Astronomy, University of Glasgow, G12 8QQ, Scotland.

Procheta Mallik is with the Dept. of Physics and Astronomy, University of Glasgow, G12 8QQ, Scotland.

M. L. McConnell is with the Space Science Center, University of New Hampshire, Durham, NH 03824, USA.

M. R. Moser was formerly with the Physikalisches Institut, University of Bern, CH-3012 Bern, Switzerland.

B. Pirard is with the Physikalisches Institut, University of Bern, CH-3012 Bern, Switzerland.

J. M. Ryan is with the Space Science Center, University of New Hampshire, Durham, NH 03824, USA.

R. S. Woolf is with the Space Science Center, University of New Hampshire, Durham, NH 03824, USA.

Neutrons in the $\mathrm{MeV}$ energy range produced in solar flares provide key information on the time evolution of the spectra of accelerated protons and ions at the flare site. They uniquely sample a wide range of the proton energy spectrum, and their numbers reflect the composition of the high-energy ions. When complemented with $\gamma$-ray data, one has indicators and measures of the proton and ion spectra from a few $\mathrm{MeV}$ up to $\mathrm{GeV}$ energies [4]. When compared to $\gamma$-rays, neutrons present the unique advantage of a continuous energy spectrum with no beraks through the entire $\mathrm{MeV}$ interval [5].

FNIT was specifically designed by an international team to cover the 1-20 MeV neutron energy range. A separate instrument, the SOlar Neutron TRACking imaging spectrometer (SONTRAC), was developed by the same team with the addition of University of Alabama - Huntsville, for higher energies (20-250 MeV) [6]. Since low energy solar neutrons do not survive in significant numbers by the time they reach the Earth, energies below $\sim 20 \mathrm{MeV}$ are hardly accessible at 1 astronomical unit (AU). Therefore, customized neutron spectrometers have to be operated on missions to the innermost heliosphere.

The need to measure neutrons below $10 \mathrm{MeV}$ has been recognized by NASA as one of the solar physics objectives. 
Considering the need to take measurements in close proximity to the Sun, the Solar Sentinels program provides an ideal platform for the FNIT detector. The present design of the Solar Orbiter spacecraft also includes in its instrument complement a detector for low $\mathrm{MeV}$ neutrons, with FNIT being a potential candidate to fulfill this mission requirement as well.

\section{SPECIAL NUCLEAR MATERIAL}

One of the critical gaps in homeland security is the inability to efficiently detect and identify kilogram-size samples of SNM. These materials, uranium and transuranics, emit neutrons via spontaneous or induced fission. Unlike other forms of radiation produced by SNM (e.g. $\gamma$-rays), copious and penetrating neutron emission is unique to fissionable material. Neutron detection, therefore, is of particular importance for SNM identification for security and proliferation deterrence.

Current detection systems often do not have sufficient sensitivity to detect problematic and dangerous amounts of fissionable material. For example, one kilogram of weapon grade plutonium (WGP) emits $6 \times 10^{4}$ neutrons/second. At a distance of $10 \mathrm{~m}$, this translates into a flux of $5 \times 10^{-3}$ neutrons $/ \mathrm{cm}^{2} \mathrm{~s}$, while the sea-level atmospheric neutron flux in the range 1-5 MeV measured by [7] is $\sim 10^{-2}$ neutrons $/ \mathrm{cm}^{2} \mathrm{~s}$. In other words, and unless active interrogation methods are used, the flux from the WGP sample is overshadowed by background neutrons at a distance of only several meters in a detector lacking imaging capability. As a consequence, the ability to search for clandestine amounts of SNM currently represents a major security shortcoming.

The energy spectrum of fission neutrons from ${ }^{239} \mathrm{Pu}$ follows the Watt distribution, peaking at $1 \mathrm{MeV}$ and with a mean value of $\sim 2 \mathrm{MeV}$ [8], [9]. In order to identify Pu samples, it is crucial to possess a detector with both imaging capability and an energy threshold of the order of $1 \mathrm{MeV}$ or less. Both these requirements are satisfied by the FNIT design.

\section{Neutron Detection AND IMAGING}

Because they are electrically neutral, neutrons must be detected using indirect means. The preferred method at $\mathrm{MeV}$ energies takes advantage of the fact that the n-p elastic scattering cross section is large. The recoil proton from an $n-p$ scatter is a highly ionizing particle and is easily detected with a suitable instrument. To employ this technique in its full potential, it is advantageous to have neutrons scatter off protons rather than heavier nuclei. Therefore, an ideal material, acting both as neutron scatterer and recoil proton detector, is organic scintillator [10].

To perform imaging, it is necessary for the neutron to undergo several (at least two) n-p scatters in the detector. One must be able to follow the path of the neutron, measuring the location, time and energy deposits of each n-p interaction.

Consider the case shown in Fig. 1. A neutron, whose incident direction is unknown, undergoes two n-p scatters. By measuring the coordinates and time difference of the two interactions, one can determine the energy and direction of the

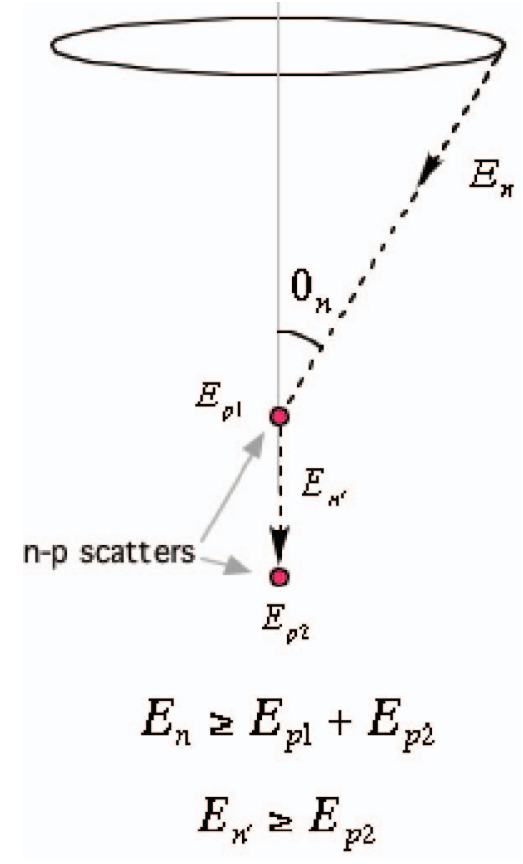

Fig. 1. Double elastic n-p scattering. The schematic displays the basic kinematics of event reconstruction. If the kinetic energy of the incident neutron is determined, the neutron arrival direction can be restricted to lie on the mantle of a cone. An "event circle", such as the one shown at the top of this figure, can then be drawn.

scattered neutron. By measuring the energy of the first recoil proton, one can then compute the energy of the incident neutron. The neutron scatter angle, $\theta_{\mathrm{n}}$, is given by:

$$
\sin ^{2} \theta_{\mathrm{n}}=\frac{E_{p 1}}{E_{n}}
$$

where $E_{\mathrm{p} 1}$ and $E_{\mathrm{n}}$ are the energies of the first recoil proton and the incident neutron, respectively. Once $E_{p 1}$ and $E_{n}$ are known, one can determine $\theta_{\mathrm{n}}$. In general, if the vector direction of the first recoil proton were known, one could determine the exact direction of the incident neutron. However, at FNIT energies, recoil proton tracks are too short for their orientation to be determined and one can only measure the proton's kinetic energy. With this information, knowing the direction of the recoil neutron, one can constrain the incident neutron's track to lie on the mantle of a cone.

By projecting the cone onto an image plane, one can then define an "event circle" for each event, as shown in Fig. 1. Finally, from the intersection of several event circles, it is possible to obtain an image of the neutron source. A very similar technique is employed for $\gamma$-ray imaging in Compton telescopes. Namely, event circles from a source intersect at a specific point, while the imaging of an isotropic background produces circles with isotropically distributed intersections. The full potential of this method was demonstrated in the past in the imaging of 20-80 MeV solar neutrons [11] and is presently being refined to image neutron sources in the 1-20 $\mathrm{MeV}$ range from FNIT measurements. 


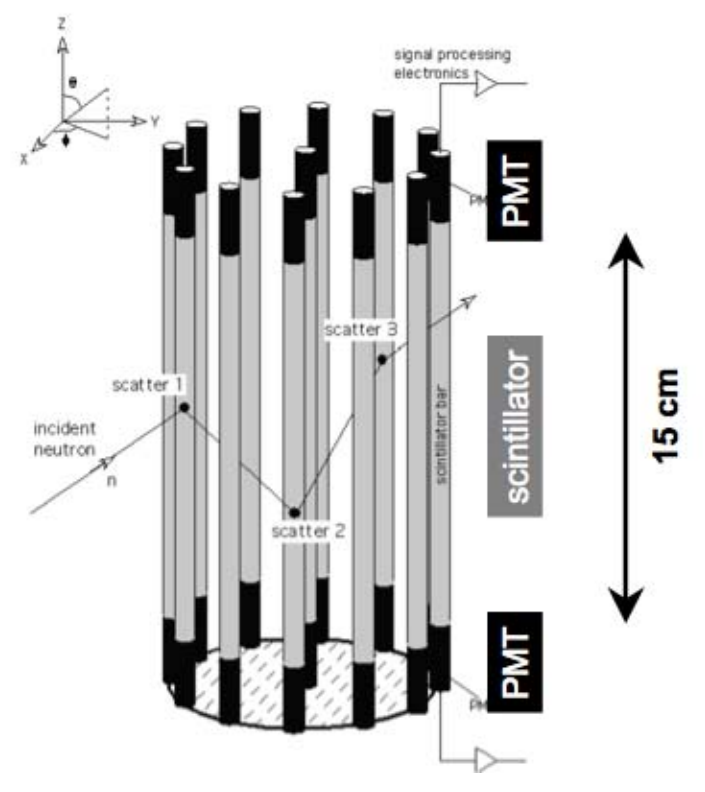

Fig. 2. Schematic view of the layout of the full FNIT detector concept. Liquid scintillator bars are arranged in a cylindrically symmetric pattern and every bar is coupled to two PMTs, one at each extremity.

\section{The FAST NeUtron IMAging Telescope}

In its present configuration, the FNIT detector design consists of a bundle of liquid scintillator bars, arranged in a cylindrically symmetric pattern, as shown in Fig. 2. Each bar is $15 \mathrm{~cm}$ long and has two photomultiplier tubes (PMTs) coupled directly to the scintillator fluid, one at each extremity of the bar. The circular bundle is also $15 \mathrm{~cm}$ in diameter, making FNIT a compact and lightweight detector with a full $360^{\circ}$ field of view. The number and size of the bars have been chosen for FNIT to fit into the expected mass and size budget of the planned Solar Sentinel mission, but the detector concept is based on a modular design and the number of bars, as well as their length, can be in principle increased as necessary, depending on the specific application.

Each bar consists of a thin aluminum vessel, $1.5 \mathrm{~cm}$ in diameter, that hosts the liquid scintillator. Organic scintillator is being used due to its hydrogen content, hydrogen nuclei providing the scatter centers for neutron elastic scattering. Liquid scintillator was chosen over plastic since it provides the unique advantage of pulse shape discrimination (PSD) between neutrons and $\gamma$-rays [10]. The $1.5 \mathrm{~cm}$ diameter of the scintillator bar is a compromise used to maximize the probability of having one elastic n-p scattering take place, while minimizing the chance of having more than one such scattering occur in the same bar [3].

The science model prototype of FNIT, consisting of three liquid scintillator bars, was recently assembled at $\mathrm{UNH}$ and is displayed in Fig. 3. The aluminum vessels were filled with either NE-213A or BC-519 liquid scintillator [12] and a thin layer of diffuse reflective white paint was deposited on the inner surface of the aluminum containers for interior coating.

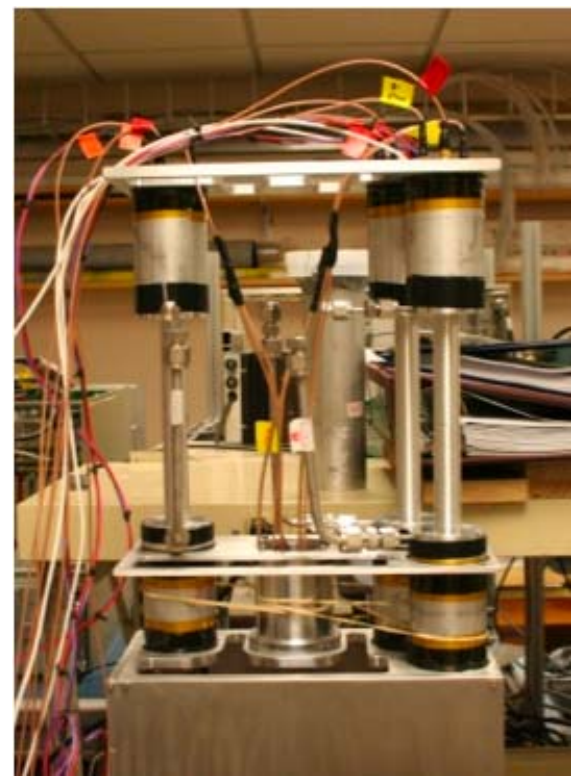

Fig. 3. The FNIT prototype detector, recently assembled at $\mathrm{UNH}$, consists of three liquid scintillator bars cased in Al vessels, with two PMTs coupled to each bar.

Two Hamamatsu R7400 PMTs [13] were coupled directly to each bar. Custom-designed preamplifiers were attached to the PMTs and the PMT pulse signals were processed through a combination of NIM and VME electronic modules.

Both PMT pulse height and particle time-of-flight (ToF) were recorded for each event. PSD was implemented by measuring the pulse time width of each PMT signal. The prototype was assembled to be operated in either the doublecoincidence mode (trigger logic requiring the coincident signals from two scintillator bars) or in the singles mode (trigger signal being provided by the pulse above threshold from at least one bar). A Labview-based software package was custom-developed for real-time data acquisition and processing.

A Monte Carlo simulation code, based on the GEANT4 package [14], was developed to model the response of the FNIT detector and optimize its design layout. Fig. 4 displays the efficiency of the full FNIT detector design as a function of energy, obtained from Monte Carlo data. These simulations did not include a low energy cutoff, which arises from limited photoelectron statistics, that was later evaluated from experimental data. Results in Fig. 4 are realistic for neutron energies above $\sim 1 \mathrm{MeV}$, where a relatively uniform and energy-independent efficiency of $\sim 5 \%$ is achieved, but the actual efficiency is expected do decrease rapidly for neutron energies below the $1 \mathrm{MeV}$ threshold.

\section{FNIT PRototype PERFormanCE}

The FNIT prototype detector described in the previous section was calibrated and its performance assessed using several different $\beta, \gamma$ and neutron lab sources, specifically: ${ }^{90} \mathrm{Sr},{ }^{109} \mathrm{Cd},{ }^{57} \mathrm{Co},{ }^{137} \mathrm{Cs},{ }^{60} \mathrm{Co}$ and an $\mathrm{Am}-\mathrm{Be}$ neutron source. 


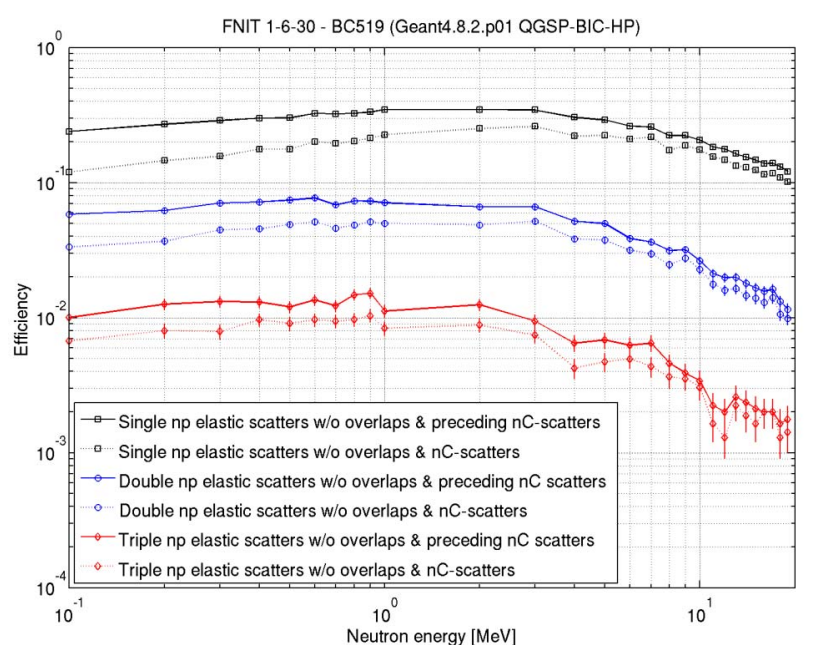

Fig. 4. Neutron detection efficiency of the full FNIT detector, evaluated through Monte Carlo simulations. At a minimum, two elastic n-p scatters are required for neutron identification, with no signal degradation from $\mathrm{n}-\mathrm{C}$ scattering taking place. Therefore, the efficiency of the FNIT instrument is best represented by the dashed blue curve, fourth from the top.

The calibration process included the evaluation of the energy threshold (Fig. 5), the assessment of position sensitivity along the liquid scintillator bar from the PMT pulse heights recorded at both extremities and an evaluation of the position resolution, the estimate of the ToF resolution measured between liquid scintillator bars, the energy calibration of the PMT response and the evaluation of the energy resolution from $90^{\circ}$ Compton scatter ${ }^{137} \mathrm{Cs} \gamma$-rays (Fig. 6), and finally the validation of the PSD capabilities of the FNIT prototype with the Am-Be neutron source. Results from these calibration runs can be summarized as follows:

- energy threshold $\mathrm{E}_{\text {thr }} \leq 20 \mathrm{keV}_{\text {ee }}$

- position resolution along the bar $\delta z \approx 0.9 \mathrm{~cm}$

- ToF resolution $\delta \mathrm{t} \approx 1.3 \mathrm{~ns}$

- energy resolution ( $374 \mathrm{keV} \gamma$-rays) $\delta \mathrm{E} / \mathrm{E} \approx 10 \%$

It should be noted that the light yield from protons at $\mathrm{keV}$ energies in liquid scintillator is an order of magnitude smaller than the light yield of electrons [12]. In other words, an electron-equivalent energy threshold of $20 \mathrm{keV}_{\text {ee }}$ corresponds to an effective threshold of a few hundred $\mathrm{keV}$ in the case of protons. Furthermore, a minimum of two scatter protons are needed to detect a neutron and therefore the neutron energy threshold of FNIT can be set at slightly less than $1 \mathrm{MeV}$. As far as energy resolution, the energy of a neutron has to be reconstructed from several measured parameters, not just from the PMT pulse heights of one liquid scintillator bar. Therefore, the $\delta \mathrm{E} / \mathrm{E}$ figure quoted previously is not representative of the neutron energy resolution of FNIT.

The FNIT prototype detector was eventually irradiated at the neutron beam at Crocker Nuclear Laboratory, UC Davis. A full description of this facility is provided by [15]. The FNIT prototype was operated at trigger rates of up to $10 \mathrm{kHz}$ and run times of up to 24 hours with no observed flaw in its performance. Data were collected at five different neutron

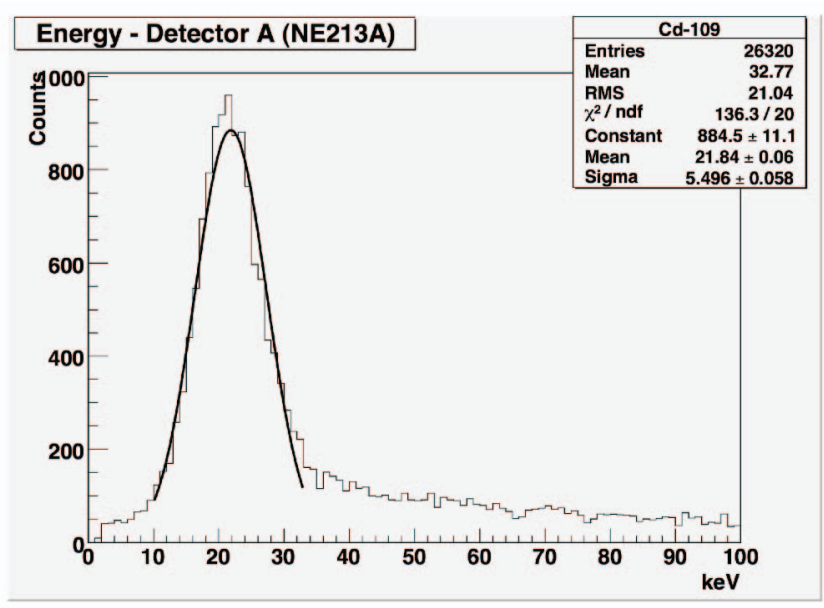

Fig. 5. Photopeak of ${ }^{109} \mathrm{Cd} \gamma$-rays in the FNIT prototype liquid scintillator bars. The spread in this distribution arises mainly from the limited number of photoelectrons. From Poisson statistics, it can be estimated that the $20 \mathrm{keV}_{\text {ee }}$ peak that appears in this figure was generated by a total of 10 to 20 photoelectrons per event.

beam energies, namely with $1,2,5,10$ and $18 \mathrm{MeV}$ neutrons, and with varying orientations of the prototype detector, to account for the various possible scatter angles of the full FNIT instrument. From the recorded coordinates and recoil proton energies of two elastic n-p scatters and the time of flight of the scatter neutron between two liquid scintillator bars we reconstructed the energies of the beam neutrons on an eventby-event basis. Results are presented in Fig. 7. An average neutron energy resolution of $\delta \mathrm{E} / \mathrm{E} \approx 20 \%$ was achieved, this value improving slightly with energy. This resolution figure is most likely an overestimate, due to the fact that beam neutrons were generated by monoenergetic protons incident on a $\mathrm{Li}$ target, the thickness of the target resulting in a non-negligible spread in the energy of the beam neutrons.

Finally, the FNIT prototype detector was exposed to a ${ }^{239} \mathrm{Pu}$ source at PNNL and its ability to detect SNM sources was thus demonstrated. A description of this measurement campaign and its results appear elsewhere in these proceedings [16].

\section{IMAGING}

A first estimate of the imaging capabilities of FNIT is represented by the value of the angular resolution measurement (ARM) [17] of the prototype detector. The value of the ARM was obtained from neutron beam data, by reconstructing the angle of the incident neutron $\theta_{\mathrm{n}}$ (see Fig. 1) and taking the difference between reconstructed angle and known direction (i.e. the known location of the neutron beam port). Results are presented in Fig. 8. An average ARM resolution of $\delta \theta \approx 5^{\circ}$ was observed, this value improving slightly with energy.

A full algorithm to reconstruct the image of the neutron source is presently being developed. The preliminary version was tested by imaging the neutron beam port at Crocker Laboratory. The procedure is described in Section IV and all 


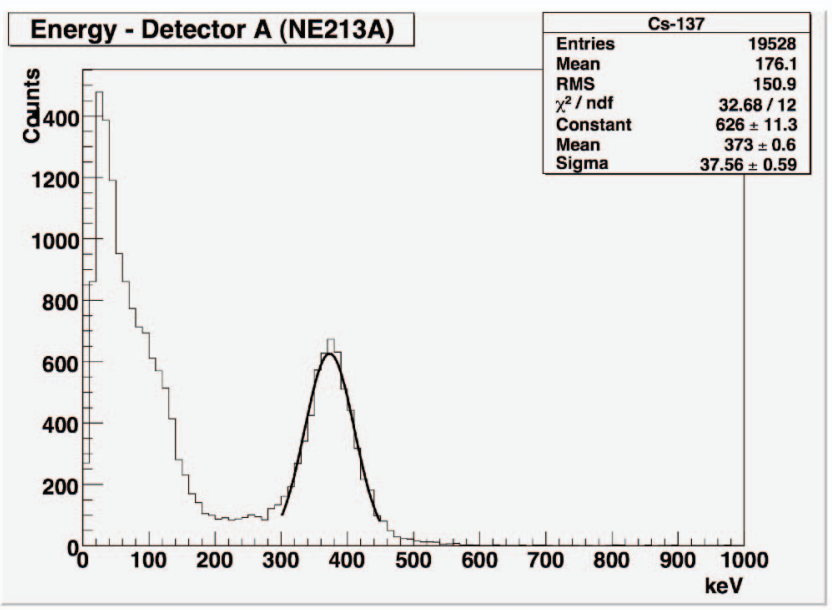

Fig. 6. Energy resolution of the FNIT liquid scintillator bar. This figure was obtained from ${ }^{137} \mathrm{Cs} \gamma$-rays, Compton-scattering in the scintillator bar at a $90^{\circ}$ angle. The central energy is $374 \mathrm{keV}$. The $\delta \mathrm{E} / \mathrm{E}$ value from this distribution is representative of resolutions for thowe energies which can be obtained from a single measured parameter. However, multiple measurements are needed to reconstruct the energy of each neutron event.

the necessary parameters for each detected neutron were recorded in the beam test data. Neutrons were separated from background $\gamma$-ray events Compton-scattering between liquid scintillator bars both from the measured ToF and by PSD. Event circles were then reconstructed on an event-by-event basis. A major shortcoming of the FNIT prototype detector when compared to the full FNIT instrument is the limited number of scintillator bars, which translates into a limited number of available scatter directions for the recoil neutrons. This in turn causes most event circles to nearly overlap each other, making it difficult to identify a clear intersection point.

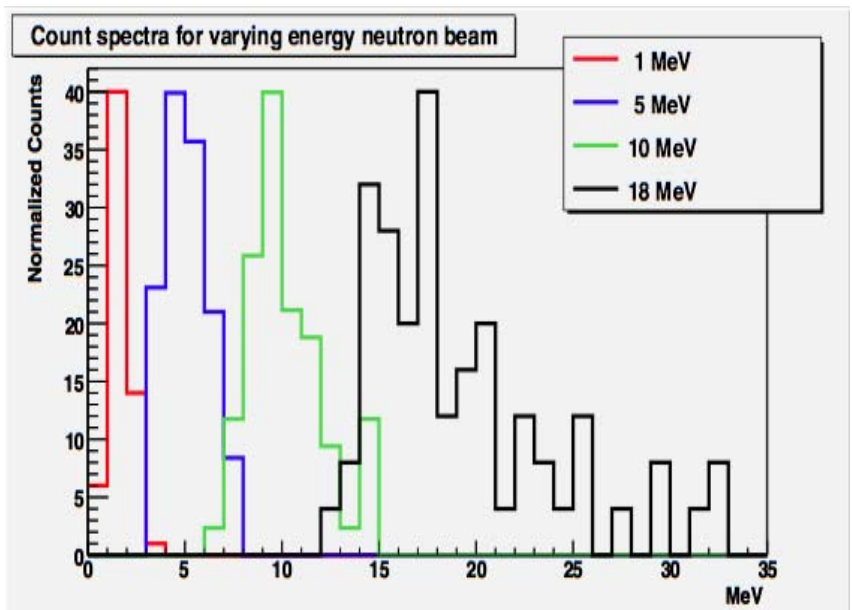

Fig. 7. Distributions of the neutron energies measured by the FNIT prototype instrument when exposed to the neutron beam at Crocker Lab, UC Davis. Four neutron beam energies were used to create this plot: 1,5,10 and $20 \mathrm{MeV}$ beam neutrons. An average energy resolution of $\delta \mathrm{E} / \mathrm{E} \approx 20 \%$ for neutron events was achieved.

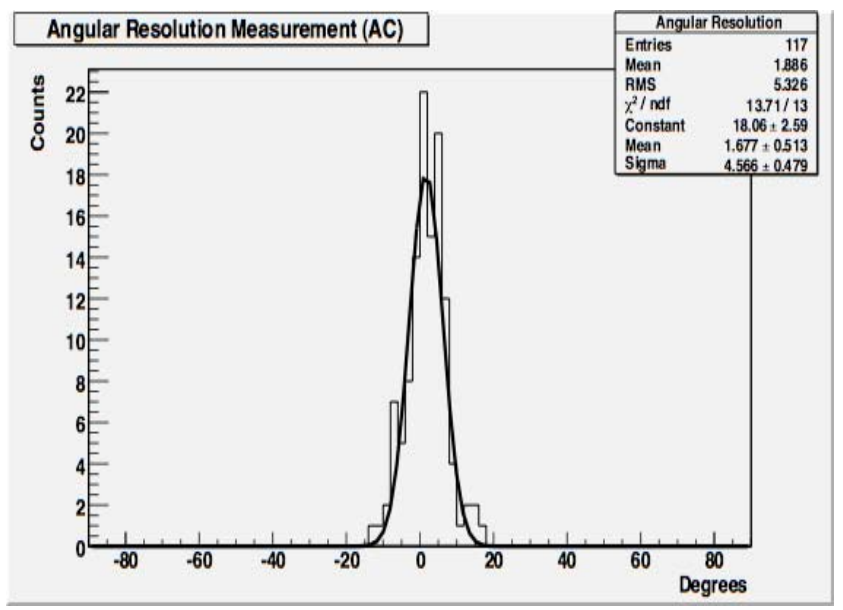

Fig. 8. ARM distribution for $10 \mathrm{MeV}$ neutrons in the FNIT prototype detector, obtained from neutron accelerator beam data. Each entry in this histogram represents the difference between the reconstructed direction angle of the incident neutron $\theta_{\mathrm{n}}$ and the actual angle of arrival of the neutron, obtained from the known location of the port of the neutron beam.

To accurately model the potential of the full FNIT detector, where multiple scatter directions become possible, we overlapped the circles resulting from data taken with the prototype detector at different orientation angles with respect to the neutron beam, ranging from $0^{\circ}$ to $90^{\circ}$. Results obtained are presented in Fig. 9. A clear intersection of event circles can be identified and the location of the neutron source clearly marked. This figure is very encouraging, although the resolution achieved so far, of the order of $30^{\circ}$, leaves plenty of room for improvement.

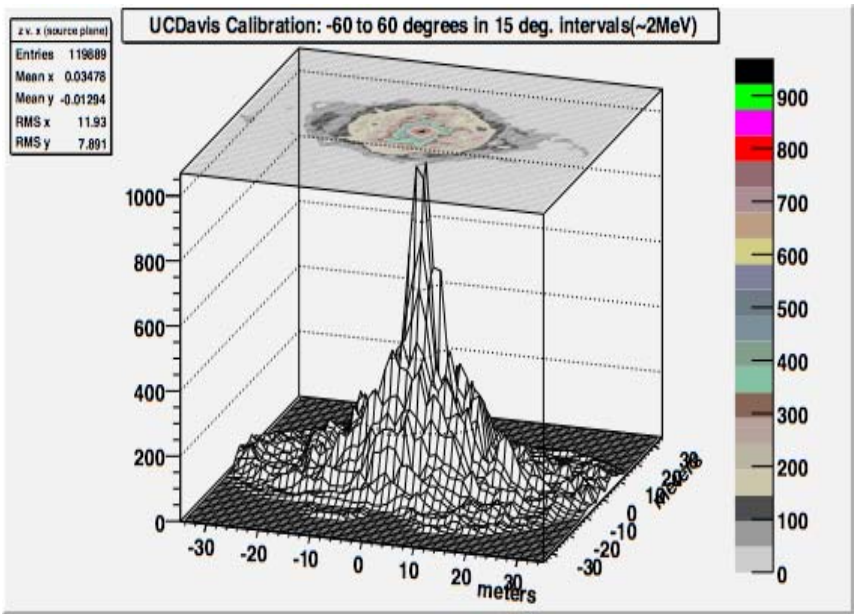

Fig. 9. Neutron source imaging with the FNIT detector prototype. This image was obtained from neutron beam data, by reconstructing the event circles of each neutron and projecting them onto the image plane (the horizontal plane at the bottom of the figure). The height of each bin in the histogram represents the number of circles that intersect at that coordinate location in the image plane. 


\section{CONCLUSIONS}

We presented a summary of the present status of the FNIT prototype instrument and a description of its design optimization and performance capabilities. FNIT R\&D efforts are presently at an advanced stage. A full science model prototype detector has been assembled and its performance assessed by exposing FNIT to an accelerator neutron beam and by irradiating it with a ${ }^{239} \mathrm{Pu}$ source. The imaging algorithm is still being developed, but preliminary results are very encouraging.

\section{ACKNOWLEDGMENT}

The authors would like to thank Dr. Carlos Castaneda of UC Davis and the staff of Crocker Lab for their assistance and support during the FNIT neutron beam tests. The authors are also grateful to Dr. Mitchell L. Woodring of PNNL for arranging and organizing the measurements with a ${ }^{239} \mathrm{Pu}$ source.

\section{REFERENCES}

[1] http://lws.gsfc.nasa.gov/missions/sentinels/sentinels.htm

[2] http://www.esa.int/science/solarorbiter

[3] U. Bravar, P. J. Bruillard, E. O. Flückiger, J. R. Macri, M. L. McConnell, M. R. Moser, J. M. Ryan, and R. S. Woolf, "Design and Testing of a Position-Sensitive Plastic Scintillator Detector for Fast Neutron Imaging," IEEE Trans. Nucl. Sci., vol. 53, no. 6, pp. 38943903, 2006.

[4] J. A. Lockwood, H. Debrunner, and J. M. Ryan, "The Relationship Between Solar Flare Gamma-Ray Emission and Neutron Production," Solar Phys., vol. 173, pp.151-176, 1997.

[5] J. M. Ryan, "Long Duration Solar Gamma-Ray Flares," Space Sci. Rev., vol. 93, pp. 581-610, 2000.

[6] R. S. Miller, J. R. Macri, M. L. McConnell, J. M. Ryan, E. Flückiger, and L. Desorgher, "SONTRAC: An imaging spectrometer for $\mathrm{MeV}$ neutrons," Nucl. Inst. Meth., vol. A505, pp. 36-40, 2003.

[7] M. R. Moser, J. M. Ryan, L. Desorgher, and E. O. Flückiger, "Atmospheric neutron measurements in the $10-170 \mathrm{MeV}$ range," in Proc. $29^{\text {th }}$ Int. Cosmic Ray Conf., 2005, vol. SH35, pp. 101-104.

[8] N. Nereson, "Fission Neutron Spectrum of $\mathrm{Pu}^{239}$," Phys. Rev., vol. 88, pp. 823-824, 1952.

[9] B. E. Watt, "Energy Spectrum of Neutrons from Thermal Fission of U ${ }^{235}$," Phys. Rev., vol. 87, pp. 1037-1041, 1952.

[10] G. F. Knoll, Radiation Detection and Measurement, 3rd ed., New York: Wiley, 2000, pp. 553-563.

[11] H. Debrunner, "Neutrons fron the 15 June 1991 Solar Flare," Proc. 23rd Int. Cosmic Ray Conf., vol. 3, pp. 115-118, 1993.

[12] http://www.detectors.saint-gobain.com/

[13] http://www.hamamatsu.com

[14] S. Agostinelli, et al., "GEANT4: A Simulation Toolkit," Nucl. Inst. Meth., vol. A506, pp. 250-303, 2003.

[15] J. A. Jungerman, and F. P. Brady, "A Medium-Energy Neutron Facility," Nucl. Inst. Meth., vol. 89, pp. 167-172, 1970.

[16] J. R. Macri, U. Bravar, J. S. Legere, P. Mallik, B. Pirard, J. M. Ryan, and R. S. Woolf, "The Fast Neutron Imaging Telescope (FNIT) Hardware Development and Prototype Testing," N10-3, these proceedings.

[17] V. Schonfelder, et al., "The GRO / COMPTEL Mission - Instrument Description and Scientific Objectives," in Data Analysis in Astronomy $I V$, New York: Plenum Press, 1992, pp. 185-200. 